\title{
Isolation of Bacillus From the Gut of Bombus terrestris and Its Correlation in Queen Mating
}

\author{
Kai Li ${ }^{1, \dagger}$, Liuhao Wang ${ }^{1,2, \uparrow}$, Zhengyi Zhang ${ }^{1}$, Yulong Guo ${ }^{1}$, Jun Guo ${ }^{3}$, Nasar Amjad ${ }^{4}$, Hira Mannan Shaikh, \\ $\mathrm{Jie} \mathrm{Wu}^{1} \& \mathrm{Jilian}^{\mathrm{Li}} \mathrm{i}^{1}$ \\ ${ }^{1}$ Key Laboratory of Pollinating Insect Biology of the Ministry of Agriculture, Institute of Apicultural Research, \\ Chinese Academy of Agricultural Science, Beijing, China \\ ${ }^{2}$ College of Resources and Environmental Sciences, Henan Institute of Science and Technology, Xinxiang, Henan, \\ China \\ ${ }^{3}$ Faculty of Life Science and Technology, Kunming University of Science and Technology, Kunming, Yunnan, \\ China \\ ${ }^{4}$ Institute of Horticultural Sciences, University of Agriculture, Faisalabad, Pakistan \\ ${ }^{5}$ Department of Entomology, Faculty of Crop Protection, Sindh Agriculture University Tandojam, Sindh, Pakistan \\ Correspondence: Jilian Li, Key Laboratory of Pollinating Insect Biology of the Ministry of Agriculture, Institute of \\ Apicultural Research, Chinese Academy of Agricultural Science, Beijing, China. E-mail: \\ bumblebeelj1@hotmail.com
}

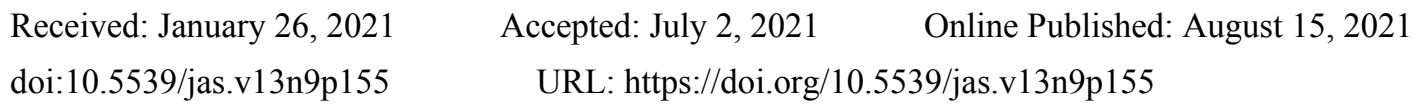

$\dagger$ These authors contributed equally to this work.

\begin{abstract}
The gut of bumblebees harbors bacteria that play a crucial role in physiology, nutrition, and health. The mating rate is important for the reproductive activity of a colony; however, few studies have investigated the relationship between mating and gut bacteria. In this study, bacterial functions were identified in the intestinal tract of bumblebees, and biochemical identification and screening were performed using genetic detection technology. By isolating and identifying bacteria, we obtained a single strain and fed it to queens. The results indicated that Bacillus cereus and Bacillus pumilus are present in the gut. The queen mating rates were $48.89 \%$ at the period of 4 days and higher than $28.89 \%$ mating rates of the control group $(\mathrm{P}<0.05)$, and the latency time were $16.90 \mathrm{~min}$ (from entering the mating cage to mating success) and decreased than control $(\mathrm{P}<0.05)$ which was 28.20 min. This finding confirmed that Bacillus was important in Bombus terrestris mating.
\end{abstract}

Keywords: mating, gut bacteria, queen bee, isolation and identification, Bacillus

\section{Introduction}

Bumblebees are important pollinators in natural and agricultural ecosystems, as well as playing a significant role in maintaining plant diversity and ecological balance (Gallai, 2009; Williams, 2009). Additionally, their potential economic value has been recognized for a long time. Their tongues are generally longer than honeybees, so they are better at pollinating flowers with deep corollas. Furthermore, the dense hairs on their bodies allow efficient pollen transfer from flower to flower, and they can also sonicate ("buzz pollination") wildflowers and crops (including tomato) whose flowers shed pollen through apical pores. Thus, some bumblebees have become commercially bred species, notably for greenhouse pollination of many crops (Velthuis \& van Doorn, 2006; Dasgan et al., 2004).

In 1912, the first monograph on artificial bumblebee rearing was published, thus starting a new process of artificial bumblebee breeding (Sladen, 1989). Chinese native bumblebees (post hibernating queens) have been reared in the laboratory for scientific studies of social behavior, biology and pollination since 1996, and remarkable progress of Bumblebees rearing techniques and pollination in greenhouses has been made (An, 2010). Additionally, mating behavior is significant to the development of colonies (Salvarrey, 2013). Therefore, for bumblebees rearing 
techniques, the successful mating of queens is a crucial step. Unmated queens cannot survive after hibernation; only the successfully mated queens can survive and reproduce colonies (Cao, 2014; Holland, 2013).

Tasei et al. (1998) reported that the sperm vitality in the vas deferens and seminal vesicles of bumblebee adult males in Europe starts to decline 13 days after emergence, an essential time for male bees to mate successfully with queens. In artificially controlled mating cages, the optimal mating age of queen bees is 8-9 days, that of male bees is 11-12 days, and the ratio of the queen and male bees is 1:1.5 (An, 2004; Wu et al., 2005). The mating situation of artificial bumblebee breeding is mainly affected by the sexual maturity of bumblebees and the external environment, including light, temperature, humidity, and health (Thomas, 2005; Amin, 2012).

The bumblebee harbors a simple yet specialized gut microbiota (Kwong et al., 2013; Killer et al., 2014; Praet et al., 2017), which plays an essential role in host food digestion and absorption, enhancing the host's immunity, and resisting pathogen infection (Li et al., 2015; Kwong et al., 2017). Studies have shown that there is a close, however, very complicated relationship between the host and its intestinal flora. Many gut bacteria have a probiotic effect on the host. They can induce the host to produce antibacterial peptides and defensins (Evans, 2004; Cariveau, 2014). Bacillus subtilis can inhibit bee funga Ascosphaera apis and play an essential role in the prevention of Nosema sp. (Liu et al., 2016). Various Bacillus bacteria have inhibitory effects on Paenibacillus larvae that cause American foulbrood (Sabate, 2014). Additionally, studies have shown that bumblebee intestinal bacteria exert a defensive particular impact on the infection of Crithidia bombi (Koch \& Schmid-Hempel, 2011, 2012).

Moreover, the gut microbiota plays a vital role in the healthy reproduction of many insects, gut bacteria could influence the mating active through epidermal sex pheromone in Drosophila melanogaster. At the same time, in mouse intestinal symbiotic bacteria could impact sexual maturity time (Vanbelle, 1990; Benvacoub, 2003; Weger et al., 2018; Sharon et al., 2010). Our previous study showed a significant difference in the diversity and composition of the gut microbiota communities in queens of Bombus lantschouensis across different physiological states, and Bacillus is a relatively dominant bacterial genus in mated queens (Wang et al., 2019). However, few studies have examined how the gut microbiota responds to external changes, as well these changes affect the interaction between the host and the external environment (Kong et al., 2014).

In order to understand the complex interplay between the microbiome and queens that mated successfully. We isolated Bacillus cereus and Bacillus pumilus from the gut of queens that mated successfully and fed Bacillus cereus and Bacillus pumilus to newly emerging queen samples to observe the effect of Bacillus on the mating rates of queens. Our study is the first to explore the impact of the gut microbiome on the mating of bumblebee queens. It will provide useful information for future studies of the function of gut bacteria in queen mating and reproduction.

\section{Materials and Methods}

\subsection{Sample Collection}

Queens of Bombus terrestris (Linnaeus) (Hymenoptera: Apidae) were collected from the Institute of Apicultural Research, CAAS, China. The colonies were reared in the dark room at a temperature of $27 \pm 1{ }^{\circ} \mathrm{C}$ and relative humidity of $50-60 \%$. Sugar water $(1: 1 \mathrm{v} / \mathrm{v})$ and apricot (Prunus armeniaca L) pollen were provided ad libitum to Paenibacillus larvae he colonies subsequently produced 100 colonies until males and gynes (new queens) emerged.

\subsection{Isolation and Culture of Bacteria}

Three healthy mated queen bees from the bumblebee breeding room were collected and pretreated in the refrigerator at $4{ }^{\circ} \mathrm{C}$ for 5 minutes. After that, the queen was moved to an ultra-clean bench for intestinal extraction (Wang et al., 2019). The whole gut was placed in a $1.5-\mathrm{mL}$ centrifuge tube, and then $800 \mu \mathrm{L}$ of solution (Krebs-Ringer Solution) was added. Thereafter, the sample was fully ground into a homogenate, and then $100 \mu \mathrm{L}$ and $900 \mu \mathrm{L}$ of $\mathrm{ddH}_{2} \mathrm{O}$ were added and mixed with a pipette. The sample was diluted 10 times, 100 times and 1000 times, and finally coated with 1000 times diluted bacterial solution (Tryptic Soy Broth-TSB solid culture medium). The culture medium was placed in a constant-temperature incubator for $48-72 \mathrm{~h}$, and the temperature was $37 \pm 1^{\circ} \mathrm{C}$. After the strain spot appeared, strain spot was streaked and cultured purely 2-3 times. The obtained strain was subjected to Gram staining, and then the morphology of the bacteria was observed under a microscope.

We selected representative and single strains grown on the culture medium. After repeated purification for 2-3 times, we placed them in an incubator room for 2-3 days. We observed and recorded the morphological characteristics of bacterial colonies on the plate. 


\subsection{Identification of Bacteria}

Total genomic DNA was extracted using a DNA purification kit $\left(W_{i z a r d R}{ }^{@}\right.$ SV 96 Genomic DNA Purification System; (Promega) from 18 hour cultures of each isolate. DNA samples were stored at $-20{ }^{\circ} \mathrm{C}$ for PCR and further analysis. The bacterial 16S rRNA gene was amplified by PCR from total DNA using primers 27F and 1492R (Engel et al., 2013). The PCR products were electrophoresed in $1.2 \%$ agarose gels containing $0.5 \mu \mathrm{g} / \mathrm{ml}$ GoldView (GV) and visualized under UV light. Some of the PCR-amplified bands were purified and sequenced to verify their identities. The PCR products were bi-directionally sequenced, and the sequencing work was completed by Sino Geno Max (Beijing, China). The sequences obtained were BLAST analyzed and matched with valid reference sequences in the NCBI (National Center for Biotechnology Information) to determine the bacterial species types. Individual sequences of isolates that were $\geq 98 \%$ identical to each other were considered the same phylotype and were combined for analysis.

At the same time, the physiological and biochemical identification of Bacillus were performed according to Dong et al. (2001), including starch hydrolysis, VP, gelatin, nitrate, and lecithin test.

\subsection{Feeding Bacillus}

In total, 270 newly emerging queen samples were collected from the colonies of 3 Bacillus-fed groups (Bacillus + Sugar water, $5 \times 107$ copies $/ \mathrm{ml}$ (Wang et al., 2019) mixed with $50 \%(1: 1 \mathrm{v} / \mathrm{v})$ sugar) and 3 control groups without Bacillus cereus and Bacillus pumilus feeding, each group comprising 15 queens randomly distributed into 6 experimental groups ( $\mathrm{N}=15$ queens/group): 3 groups with Bacillus supplement and 3 without Bacillus supplement. Bacillus was collected from the guts of healthy mated queens and isolated on TSB medium. Then the queens were fed individually with the Bacillus liquid each day, for a period of 3, 4, and 5 days before undergoing a mating test. The concentration of the Bacillus cereus and Bacillus pumilus mixture (1:1) was $5 \times$ $10^{7}$ copies $/ \mathrm{ml}$ (Wang et al., 2019) mixed with $50 \%(1: 1 \mathrm{v} / \mathrm{v})$ sugar solution. All the tests were repeated three times.

\subsection{Effect of Bacillus on the Mating Success Rate of Queen Bees}

The queens and males of each group were placed together in $100 \times 100 \times 100 \mathrm{~cm}$ stainless steel cages with mesh and transparent walls to observe the mating success rate. During mating, the cages were illuminated with $200 \mathrm{~W}$ mercury bulbs positioned $1 \mathrm{~m}$ above the mating cages. The experiment was performed in rooms maintained at $26 \pm 1{ }^{\circ} \mathrm{C}$ with a queen to male ratio of $1: 2$ in the mating cages to determine the impact of Bacillus feeding on queen mating success. (Wu et al., 2005; An, 2004). Fifteen queens (each treatment group) and 30 sexually mature male bees of each group were placed in each mating cage. The mating experiment time was from 8:00 to 12:00 every day for one month (Peng, 2003). The latency (the time elapsed between the introduction of males and queens into the mating cage until the initiation of copulation) (Amin, 2012), mating time, and mating rate of the queen bees were recorded in the mating experiment. As the queens emerged from the cage, the sexual maturation increased until the full maturity and mating with the male bees completed the mating process. In this process, the queen maturity was proportional to the queen mating rate (Wu et al., 2005; An, 2004). We started to record the numbers of successful mated queens within 1 hour when queens and males entered the cage.

\subsection{Statistical Analysis}

SPSS software (version 23) was used to analyze the latency, mating time, and mating rate. The significant differences in the mating rate, mating time, and latency between the Bacillus cereus plus Bacillus pumilus mixture-fed queen group and control group (no feeding) were compared using analysis of GLMMs (An et al., 2012). The model used a binary logistic regression distribution and included mating status (success or failure) as the response variable, the days, and groups as fixed factors, and the number of bumble queens as a random factor. The latency and mating time were analyzed in the same way.

\section{Results}

\subsection{Morphological Identification of Bacillus}

By morphological identification, we obtained the two pure bacterial cultures named TSB-01 and TSB-11. The growth habits of the strains were all aerobic, and the morphological characteristics were regular round, showing a milky-opaque shape, wet surface, and no apparent gloss. After gram staining, the bacteria were Gram-positive, rod-shaped, medium-sized, most of them existed individually, and a few were connected to each other, similar to Bacillus spp. under the microscope (Dong, 2001; Sha, 2019). 


\subsection{Molecular Identification of Bacillus}

The 16rRNA sequence analysis revealed two different Bacillus strains, Bacillus cereus (TSB-01), which lengths is $880 \mathrm{bp}$, was $100 \%$ similar with MK648340.1. Bacillus pumilus (TSB-11), which lengths is $1406 \mathrm{bp}$, was $100 \%$ similar with MN581181.1. The sequences used in this analysis have been deposited in GenBank,containing the accession numbers: MT704413 and MT704414. To further identification the species of Bacillus, we performed physicochemical tests on the strains using physiological and biochemical tests (Figure 1). The physical and chemical properties of the two strains were identical to those reported in the literature (Chen, 2018; Dong et al., 2001). For TSB-01, starch hydrolysis, VP, gelatin, and nitrate were positive, and lecithin was positive, findings identical to that for Bacillus cereus. For TSB-11, VP, and gelatin were positive whereas starch hydrolysis, lecithin and nitrate were negative, results identical to that for Bacillus pumilus.
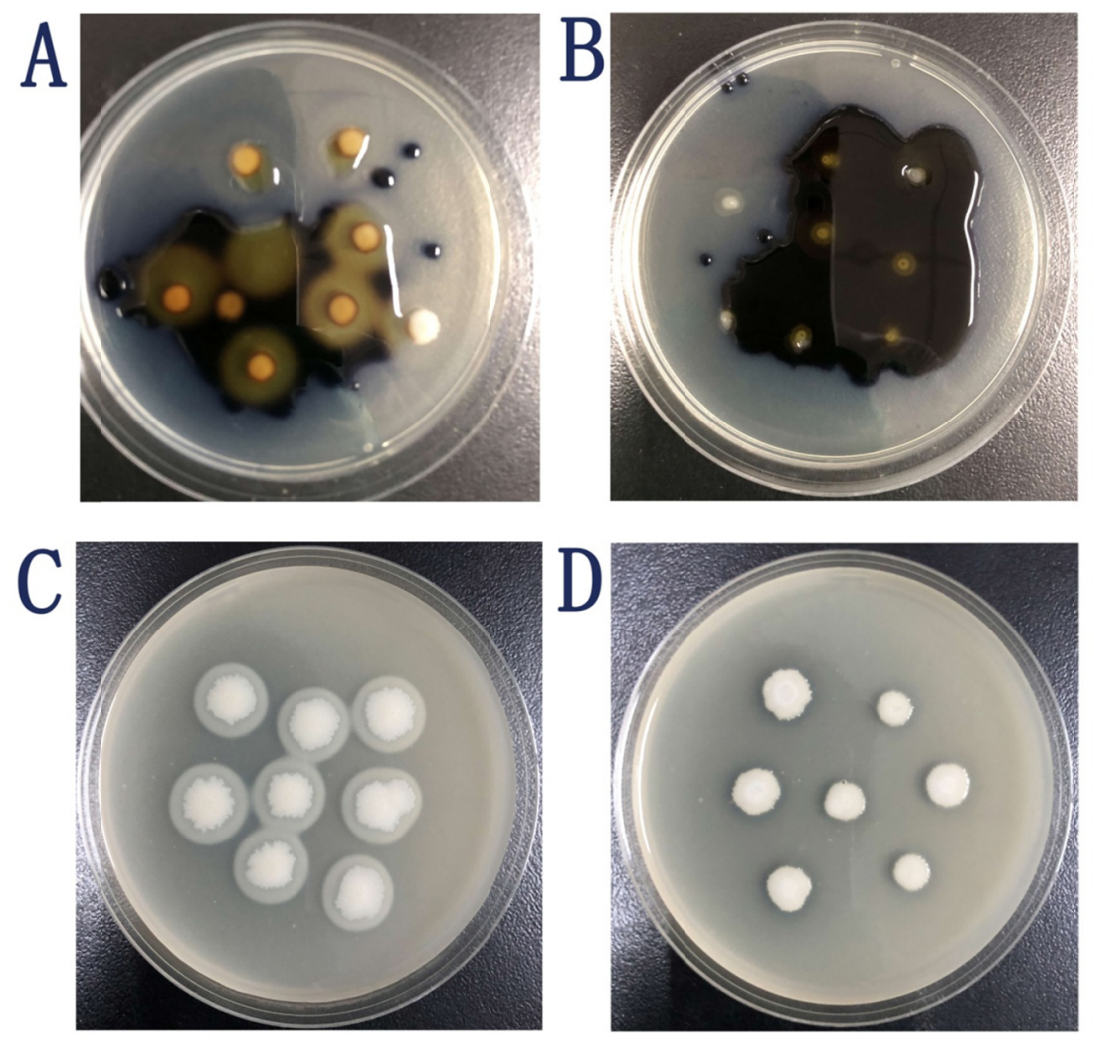

Figure 1. Physiological and biochemical tests of two Bacillus species. A: Bacillus cereus (positive starch hydrolysis test). B: Bacillus pumilus (negative starch hydrolysis test). C: Bacillus cereus (positive lecithin test). D: Bacillus pumilus (negative lecithin test)

\subsection{Effect of Bacillus on the Mating of Queen Bees}

The latency time of Bacillus-fed queen bees was significantly lower that of than the control group (queens without Bacillus feeding). Additionally, an extremely significant difference was found at 3 days $(P=0.0001$; Figure 2$)$ and 5 days $(P=0.0001$; Figure 2$)$ between the control and treatment groups. The difference between the treatment and control groups was significant at 4 days too $(P=0.033$; Figure 2$)$.

The mating rate of the Bacillus-fed group was higher than that of the control group. At 4 and 5 days, the mating rate of the feeding group was significantly higher than that of the control group ( 4 days: $P=0.033 ; 5$ days: $P=$ 0.036; Figure 2). For 3 days, although the mating rate of the feeding group was higher than that of the control group, no significant difference was found $(P=0.28$; Figure 2$)$. Additionally, for the feeding group and control, the mating rate increased as the days (age) increased: the mating rate of the feeding group was $48.89 \%$ at the period of 4 days, while that of the control group was only $28.89 \%$, with a highly significant difference between them $(P=0.033$; Figure 2). 
No significant difference was found among the, feeding, and control groups according to the mating time ( 3 days: $P=0.833 ; 4$ days: $P=0.752 ; 5$ days: $P=0.599$; Figure 2 ), which was very stable and maintained at approximately 60 minutes. Thus, no relationship was found between the mating time and degree of development of queen bees.
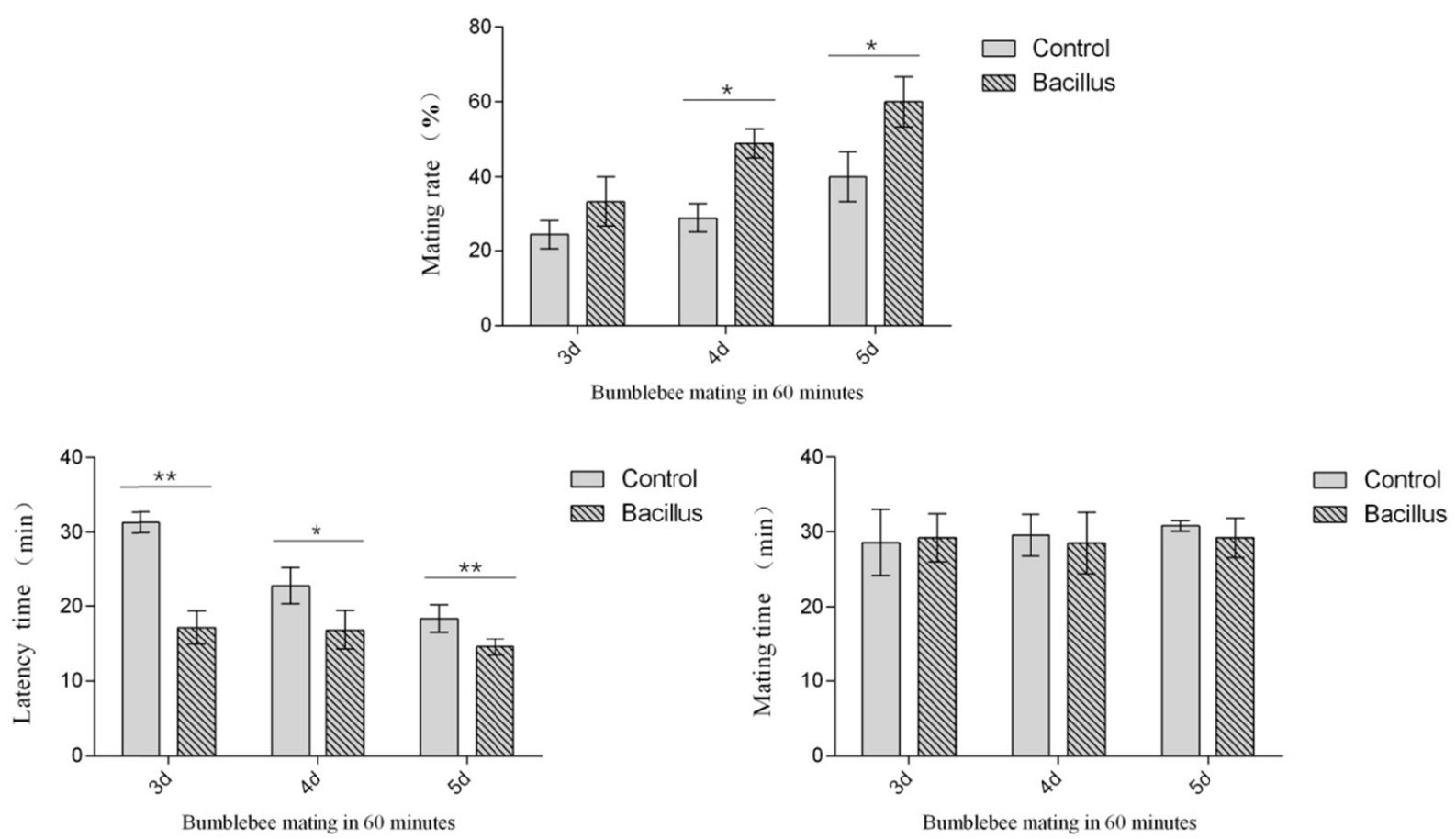

Figure 2. Comparison of the mating rate, latency time mating time and, of queen bees at different instars between the feeding and control groups. Each bar followed by "*”" indicates significant difference at the $\mathrm{P}<0.05$ level; the “**” indicates extremely significant difference at theP $<0.01$; no marks is no difference at the $\mathrm{p}>0.05$

\section{Discussion and Conclusion}

Presently, the functional research of commensal bacteria has been mainly explored molecularly, but molecular biology methods can only analyze the composition and function of microorganisms and cannot meet the needs of microbial metabolic reactions and functional verification. In this study, we obtained strains of Bacillus cereus and Bacillus pumilus using the isolation, identification, and functional verification in vitro. During the growth and development of insects, bacteria can produce some beneficial functions, including the synthesis of nutrients, digestion, and inhibition of pathogens (Koch et al., 2011; Engel et al., 2013). In this study, we isolated Bacillus cereus and Bacillus pumilus from the whole guts of bumblebees. These bacteria have different growth characteristics as well different physiological and biochemical responses. This result proves that the intestinal flora exerts different functions on the host caused by various bacteria. The microorganisms at different physiological stages may have different functions. For example, Gilliamella and Lactobacillus are dominant before the queen's mate successfully; however, after a successful mating, the predominant bacteria were Bacillus, Lactococcus, and Pseudomonadaceae in the intestine of queen bees (Wang et al., 2019).

After the two isolated strains of Bacillus were added to the bee food, the mating rate of the queen bee was significantly higher than that of the controlling queen $(\mathrm{P}<0.05)$. The latency (time from entering the mating cage to the beginning of mating) was significantly lower than that of the control group $(\mathrm{P}<0.05)$. However, no significant effect was observed on the mating time of the queen bee. Thus, Bacillus plays a specific physiological function in the queen's sexual maturity stage. Our study showed Bacillus play a role in bumblebee's reproduction the same as the other animal. Such as, studies have found that it can increase the egg production and progeny quality of female Bacillus-fed mice (Zhang, 2009). After adding Bacillus to the feed, sows showed a shorter estrus and increased appetite. It was reported that Bacillus could improve the reproductive performance of sows (Yang, 2016). After feeding Bacillus to sows, the sow intake in the experimental group increased, and the estrus interval was shortened. 
Bumblebee mating behavior is affected by many factors, including their sexual maturity and environmental conditions (An, 2004), The addition of Bacillus cereus to the feed can significantly increase the growth rate of shrimp and fish, as well as promote host body development (Tang, 2017). By promoting growth, the queen can enter sexual maturity, thereby increasing the mating rate. However, how Bacillus affects queen mating is unclear and needs further research.

The diversity of symbiotic flora in the intestinal tract of European bumblebees is very rich. Although some strains were isolated in vitro in this study, some symbiotic bacteria that could not be isolated persisted in the intestine of bumblebees. Bacillus was isolated in this study. The cause may be related to the bacteria culture conditions and choice of culture medium. The other two strains fully comprehend the physiological role and function of symbiotic bacteria in the bumblebee intestine. To isolate and culture Lactococcus and Pseudomonadaceae, selecting the appropriate culture medium and culture conditions according to their growth characteristics is necessary. In specific cases, specific nutrients must be added to promote bacterial growth. Thus, we isolated and cultured the bacteria and then analyzed the physiological and biochemical characteristics of the isolates.

In Drosophila melanogaster intestinal symbiotic bacteria could through epidermal sex pheromone influence their mating (Sharon et al., 2010). In mouse, intestinal symbiotic bacteria could impact sexual maturity time (Weger et al., 2018). This study showed that mixing B. cereus and Bacillus pumilus to feed bumblebees could increase their mating rate. A mixed preparation of Bacillus can be added to the artificial breeding system of bumblebees to increase their mating rate. However, it is not clear in which ways do Bacillus increase the mating rate on bumblebees.

Therefore, mating behavior represents an essential vital system to understand the link between bee reproduction and host-associated microbiota. The bumblebee gut bacteria likely play a role in shaping the ability of the bee to survive environmental extremes and reproduce due to long-established coevolutionary relationships between the host and microbiome members.

The results showed a significant difference in the diversity and composition of Bombus terrestris gut-isolated Bacillus, which plays a significant role in bumblebee copulation. This study provides insight into the relationship between the bacterial community and the mating of bumblebee queens. It also lays the foundation for further studies of the function of gut microbiota in the reproductive success of bumblebee queens.

\section{References}

Amin, M. R., Bussière, L. F., \& Goulson, D. (2012). Effects of Male age and Size on Mating Success in the Bumblebee Bombus terrestris. Journal of Insect Behavior, 25(4), 362-374. https://doi.org/10.1007/ s10905-011-9306-4

An, J. D. (2004). Studies on Breeding Technology of Bumblebee (Bombus lucorum) in Captivity. Chinese Academy of Agricultural Sciences, China.

An, J. D., Huang, J. X., Williams, P. H., Wu, J., \& Zhou, B. F. (2010). Species diversity and colony characteristics of bumblebees in the Heibei region of North China. Chinese Journal of Applied Ecology, 21(6), 1542-1550.

An, S. L., Zhang, Y. H., \& Chen, Z. (2012). Analysis of binary classification repeated measurement data with GEE and GLMMs using SPSS Software. Journal of Southern Medical University, 32(12), 1777-1780.

Benyacoub, J., Czarnecki-Maulden, G. L., Cavadini, C., Sauthier, T., Anderson, R. E., Schiffrin, E. J., \& Thierry, V. D. W. (2003). Supplementation of food with Enterococcus faecium (SF68) stimulates immune functions in young dogs. The Journal of Nutrition, 133(4), 1158-1162. https://doi.org/10.1093/jn/133.4.1158

Cao, N. (2014). Life histories and energetics of bumblebee (Bombus impatiens) colonies and workers. Insectes Sociaux, 14(1), 57-72.

Cariveau, D. P., Elijan, P. J., Koch, H., Winfree, R., \& Moran, N. A. (2014). Variation in gut microbial communities and its association with pathogen infection in wild bumblebees (Bombus). ISME Journal, 8(12), 2369-79. https://doi.org/10.1038/ismej.2014.68

Chen, Y. Q., Fan, Y., Saad, A., \& Jing, X. (2018). Study on Biological Characteristics and Identification of Two Bacillus Species. Journal of Shanxi Agricultural Sciences, 46(02), 175-181.

Dasgan, H. Y., Ozdogan, A. O., \& Kaftanoglu, O. (2004). Effectiveness of bumblebee pollination in anti-frost heated tomato greenhouses in the mediterranean basin. Turkish Journal of Agriculture \& Forestry, 28(2), 73-82. 
Dong, X. Z., \& Cai, M. Y. (2001). Common bacterial system identification manual. Beijing: Science Press.

Engel, P., \& Moran, N. A. (2013). The gut microbiota of insects: diversity in structure and function. FEMS Microbiology Reviews, 37(5), 699-735. https://doi.org/10.1111/1574-6976.12025

Evans, J. D., \& Lopez, D. L. (2004). Bacterial Probiotics Induce an Immune Response in the HoneyBee (Hymenoptera: Apidae). Journal of Economic Entomology, 97(3), 752-756. https://doi.org/10.1093/jee/ 97.3.752

Gallai, N., Salles, J. M., \& Settele, J. (2009). Economic valuation of the vulnerability of world agriculture confronted with pollinator decline. Ecological Economics, 68, 810-821. https://doi.org/10.1016/j.ecolecon. 2008.06.014

Holland, J. (2013). Colony Life History in the Bumblebee Bombus terrestris: Interactions, Timing and Control. University of East Anglia, Norwich, UK.

Killer, J., Votavová, A., Valterová, I., Vlková, E., Rada, V., \& Hroncová, Z. (2014). Lactobacillus bombi sp. nov., from the digestive tract of laboratory-reared bumblebee queens (Bombus terrestris). International Journal of Systematic and Evolutionary Microbiology, 64(Pt 8), 2611-2617. https://doi.org/10.1099/ijs.0.063602-0

Koch, H., \& Schmid-Hempel, P. (2011). Socially transmitted gut microbiota protects bumblebees against an intestinal parasite. Proceedings of the National Academy of Sciences, 108(48), 19288-19292. https://doi.org/ 10.1073/pnas.1110474108

Kwong, W. K., Mancenido, A. L., \& Moran, N. A. (2017). Immune system stimulation by the native gut microbiota of honeybees. Roy Soc Open Sci, 4, 170003. https://doi.org/10.1098/rsos.170003

Kwong, W. K., \& Moran, N. A. (2013). Cultivation and characterization of the gut symbionts of honey bees and bumble bees: description of Snodgrassella alvi gen. nov., sp. nov., a member of the family Neisseriaceae of the Betaproteobacteria, and Gilliamella apicola gen. nov., sp. nov., a member of Orbaceae fam. nov., Orbales ord. nov., a sister taxon to the order 'Enterobacteriales' of the Gammaproteobacteria. Int J Syst Evol Microbiol, 63(Pt 6), 2008-2018. https://doi.org/10.1099/ijs.0.044875-0

Kwong, W. K., Engel, P., Koch, H., \& Moran, N. A. (2014). Genomics and host specialization of honeybee and bumblebee gut symbionts. Microbiology, 111(31), 11509-11514. https://doi.org/10.1073/pnas.1405838111

Li, J. L., Powell, J. E., Guo, J., Evans, J. D., Wu, J., Williams, P., ... Zhang, Z. G. (2015). Two gut community enterotypes recur in diverse bumblebee species. Curr Biol, 25, R652-653. https://doi.org/10.1016/j.cub. 2015.06.031

Parmentier, A., Meeus, I., Nieuwerburgh, F. V., Deforce, D., Vandamme, P., \& Smagghe, G. (2016). A different gut microbial community between larvae and adults of a wild bumblebee nest (Bombus pascuorum). Insect Sci, 25, 66-74. https://doi.org/10.1111/1744-7917.12381

Peng, W. J., Wu, J., Guo, Z. B., \& An, J. D. (2003). A Study on mating and influential factors of Bombus lucorum under artificial conditions. Journal of Bee, 02, 3-4.

Praet, J., Cnockaert, M., Meeus, I., Smagghe, G., \& Vandamme, P. (2017). Gilliamella intestini sp. nov., Gilliamella bombicola sp. nov., Gilliamella bombi sp. nov. and Gilliamella mensalis sp. nov.: Four novel Gilliamella species isolated from the bumblebee gut. Syst. Appl. Microbiol., 40(4), 199-204. https://doi.org/10.1016/j.syapm.2017.03.003

Sabate, D. C., Carrillo, L., \& Audisio, M. C. (2009). Inhibition of Paenibacillus larvae and Ascosphaera apis by Bacillus subtilis isolated from honeybee gut and honey sample. Res. Microbiol., 160(3), 193-199. https://doi.org/10.1016/j.resmic.2009.03.002

Salvarrey, S., Arbulo, N., Santos, E., \& Invernizzi, C. (2013). Artificial breeding of native bumblebees Bombus atratus y Bombus bellicosus (Hymenoptera, Apidae). Agrociencia, 17(2), 75-82. https://doi.org/10.31285/ AGRO.17.478

Schmid-Hempel, R., \& Schmid-Hempel, P. (2000). Female mating frequencies in Bombus spp. from central Europe. Insectes Sociaux, 47(1), 36-41. https://doi.org/10.1007/s000400050006

Sha, Y. Y., Chen, J. H., Lu, Y. N., Ma, P., \& Dai, D. Z. (2019). Isolation and identification of Bacillus cereus and observation of its effect on decomposition of various poultry feathers. Animal Husbandry \& Veterinary Medicine, 51(11), 39-43. 
Sharon, G., Segal, D., Ringo, J. M., Hefetz, A., Zilber-Rosenberg, L., \& Rosenberg, E. (2010). Commensal bacteria play a role in mating preference of Drosophila melanogaster. Proceedings of the National Academy of Sciences of the United States of America, 107(46), 20051-20056. https://doi.org/10.1073/pnas.10099 06107

Sladen, F. W. L. (1989). The Humble-bee: its life history and how to domesticate it (2nd ed., p. 273). Logaston Press. London, UK.

Tang, Y., Liu, W. L., Song, X. L., Zhang, X. J., Zhang, S. J., \& Huang, Y. (2017). Effects of dietary with Bacillus cereus on the growth rate and intestinal microflora of Litopenaeus vannamei. Journal of Fisheries of China, 41(05), 766-774.

Tasei, J. N., Moinard, C., Moreau, L., Himpens, B., \& Guyonnaud, S. (1998). Relationship between aging, mating and sperm production in captive Bombus terrestris. Journal of Apicultural Research, 37, $107-113$. https://doi.org/10.1080/00218839.1998.11100962

Thomas, I., Nigel, R., \& Lars, C. (2005). Mating preference in the commercially imported bumblebee species Bombus terrestris in Britain (Hymenoptera: Apidae). Entomologia Generalis, 8(3), 233-238.

Vanbelle, M., Teller, E., \& Focant, M. (1990). Probiotics in animal nutrition: Are view. Archives of Animal Nutrition, 40(7), 543-567. https://doi.org/10.1080/17450399009428406

Velthuis, H. H. W., \& Doorn, A. (2006). A century of advances in Bombus domestication and the economic and environmental aspects of its commercialization for pollination. Apidologie, 37, 421-451. https://doi.org/ 10.1051/apido:2006019

Wang, L. H., Wu, J., Li, K., Sadd, B. M., Guo, Y. L., Zhuang, D. H., ... Li, J. L. (2019). Dynamic changes of gut microbiota communities of bumblebee queens through important life stages. Systems, 4, e00631-19. https://doi.org/10.1128/mSystems.00631-19

Weger, B. D., Gobet, C., Yeung, J., Martin, E., Jimenez, S., Betrisey, B., ... Gachon, F. (2018). The mouse microbiome is required for sex-specific diurnal rhythms of gene expression and metabolism. Cell Metab, 29, 362-382. https://doi.org/10.1016/j.cmet.2018.09.023

Williams, P. H., \& Osborne, J. L. (2009). Bumblebee vulnerability and conservation world-wide. Apidologie, 40, 367-387. https://doi.org/10.1051/apido/2009025

Wu, J., Peng, W. J., An, J. D., Guo, Z. B., Tong, Y. M., \& Li, J. L. (2005). Rearing techniques of Bumbus lucorum. Chinese Bulletin of Entomology, 06, 717-720.

Zhang, S. Q. (2009). Effect of Bacillus subtilis Pab02 Additive on the Reproductive Function of mice. Sichuan Agricultural University, China.

\section{Copyrights}

Copyright for this article is retained by the author(s), with first publication rights granted to the journal.

This is an open-access article distributed under the terms and conditions of the Creative Commons Attribution license (http://creativecommons.org/licenses/by/4.0/). 